University of Nebraska - Lincoln

DigitalCommons@University of Nebraska - Lincoln

Faculty Publications, Department of Physics and Astronomy

Research Papers in Physics and Astronomy

4-1-2005

\title{
PHOTOMETRY OF TYPE II CEPHEIDS. II. THE SHORT-PERIOD STARS
}

\author{
Edward G. Schmidt \\ University of Nebraska-Lincoln, eschmidt1@unl.edu \\ Dale Johnston \\ University of Nebraska-Lincoln, dalej96@gmail.com \\ Shawn Langan \\ University of Nebraska-Lincoln, nuaur@hotmail.com \\ Kevin M. Lee \\ University of Nebraska-Lincoln, klee6@unl.edu
}

Follow this and additional works at: https://digitalcommons.unl.edu/physicsfacpub

Part of the Physics Commons

Schmidt, Edward G.; Johnston, Dale; Langan, Shawn; and Lee, Kevin M., "PHOTOMETRY OF TYPE II CEPHEIDS. II. THE SHORT-PERIOD STARS" (2005). Faculty Publications, Department of Physics and Astronomy. 24.

https://digitalcommons.unl.edu/physicsfacpub/24

This Article is brought to you for free and open access by the Research Papers in Physics and Astronomy at DigitalCommons@University of Nebraska - Lincoln. It has been accepted for inclusion in Faculty Publications, Department of Physics and Astronomy by an authorized administrator of DigitalCommons@University of Nebraska - Lincoln. 


\title{
PHOTOMETRY OF TYPE II CEPHEIDS. II. THE SHORT-PERIOD STARS
}

\author{
Edward G. Schmidt, Dale Johnston, Shawn Langan, and Kevin M. Lee \\ Department of Physics and Astronomy, University of Nebraska-Lincoln, Lincoln, NE 68588-0111; \\ eschmidt1@unl.edu,dalej96@bigred.unl.edu,nuaur@hotmail.com,klee6@unl.edu \\ Received 2004 September 15; accepted 2004 December 27
}

\begin{abstract}
We present 668 new photometric observations for 24 Cepheids with periods less than 3 days. Most of the stars are probable type II Cepheids, but we have included some type I Cepheids for comparison. A discussion of the Fourier parameters of the light curves leads to the conclusion that they can be used to distinguish among the several types of light curves found among the type II stars but are of limited usefulness for distinguishing between type I and type II Cepheids. The pulsational stability is investigated by searching for long-term changes in the light-curve shapes, period changes, and light-curve scatter. In terms of these parameters, the pulsation is more stable than those that were found for the long-period stars, but for each there are several stars that show unusually large effects. However, there does not seem to be any obvious difference in light-curve stability of type II Cepheids compared with type I Cepheids.
\end{abstract}

Key word: Cepheids

Online material: machine-readable table

\section{INTRODUCTION}

We have undertaken a study of the observational properties of Cepheids with the goals of arriving at a clear, empirical definition of type II Cepheids and of finding reliable ways to distinguish them from classical (or type I) Cepheids. In earlier papers we presented photometry for Cepheids with periods longer than 8 days (Schmidt et al. 2004a, hereafter Paper I) and spectroscopic observations (Schmidt et al. 2003a, 2003b, 2004b) for Cepheids of all periods and considered some of the implications. The reader should refer to Paper I for a more extended discussion of the motivation for this project.

In this paper we present and discuss our photometric data for stars with periods less than 3 days. Diethelm $(1983,1986,1990)$ has conducted extensive photometric studies of such stars in which he identified several types of light curves and obtained photometric abundances for many stars. Others have analyzed the light curves through Fourier decomposition. Some of the most extensive studies in this period range were those of Antonello \& Poretti (1986), Petersen \& Diethelm (1986), Simon (1986), Antonello et al. (1990), and Mantegazza \& Poretti (1992). The present paper expands on these papers in several ways. We have added to the number of stars with reliable Fourier parameters. This allows us to better define the behavior of the type I and type II Cepheids in the various Fourier diagrams and to address the question of the homogeneity of the type II Cepheids in this period range. The stability of pulsation has theoretical implications, as well as being a possible discriminant of type. Our observations are combined with existing data to investigate the stability of the pulsation.

\section{THE OBSERVATIONS}

We have used the General Catalogue of Variable Stars (Khopolov 1985, 1987, hereafter GCVS) and the catalog of type II Cepheids by Harris (1985) to select objects. As in Paper I we have included mostly stars that are likely to be type II Cepheids but have included some likely type I Cepheids for comparison. However, the number of the latter is limited; there are only 15 stars in the GCVS with periods less than 3 days that are classified as classical Cepheids, and only one of them has a period less than 2 days.

The observations were all made at Behlen Observatory with the $0.76 \mathrm{~m}$ telescope. The instrumentation and the procedures used in collecting and processing the data are mostly the same as those used for the observations presented in Paper I, and the reader is referred to that publication for a complete description of them. An exception to this is that in the present study a single set of comparison stars was used throughout for each variable.

The stars discussed here are listed in Table 1, where column (1) lists the names of the stars, column (2) lists their approximate periods, and column (3) gives the classifications from the GCVS. A "II" in column (4) identifies stars that appear in Table I of Harris' (1985) catalog of type II Cepheids. He selected these objects on the basis of distance from the Galactic plane. Thus, we refer to stars with "II" in column (4) as high- $Z$ stars and the remainder as low- $Z$ stars, as in Paper I. We emphasize that since there are undoubtedly type II Cepheids near the Galactic plane, this distinction is, at best, a rough guide to the type of an individual star.

Diethelm (1983) developed a scheme for classifying the morphology of light curves of short-period Cepheids. These classes have been shown to be related to the metallicities of the stars (Diethelm 1990) and to the behavior of $\mathrm{H} \alpha$ (Schmidt et al. 2003b). The light-curve types for our stars are listed in column (5). They were taken from Diethelm (1990) for stars listed there and were assigned by the authors for stars he did not include. The latter are enclosed in parentheses to distinguish them.

Information about the comparison stars is provided in columns (6)-(8). Column (6) lists the number of comparison stars used for each field. Column (7) contains the standard errors of the adopted $V$ and $R$ magnitudes of the comparison stars. Column (8) gives the number of photometric nights included in calculating the comparison star magnitudes.

Because Behlen Observatory is a low-quality photometric site, placing the comparison stars on the standard system presents difficulties. As a check, we compared our $V$ light curves 
TABLE 1

The Program Stars

\begin{tabular}{|c|c|c|c|c|c|c|c|c|c|c|}
\hline $\begin{array}{l}\text { Star } \\
(1)\end{array}$ & $\begin{array}{l}\text { Period } \\
\text { (days) } \\
\text { (2) }\end{array}$ & $\begin{array}{l}\text { GCVS } \\
\text { Class } \\
(3)\end{array}$ & $\begin{array}{l}\text { Harris } \\
\text { Class } \\
(4)\end{array}$ & $\begin{array}{l}\text { Diethelm } \\
\text { L.C. Type } \\
\text { (5) }\end{array}$ & $\begin{array}{l}n_{c} \\
(6)\end{array}$ & $\begin{array}{c}\sigma_{V}, \sigma_{R} \\
(\mathrm{mag}) \\
(7)\end{array}$ & $\begin{array}{l}n_{n} \\
(8)\end{array}$ & $\begin{array}{c}\text { HJD-2,400,000 } \\
\text { (9) }\end{array}$ & $\begin{array}{c}N \\
(10)\end{array}$ & $\begin{array}{c}\text { Source } \\
\text { of Phot. } \\
\text { (11) }\end{array}$ \\
\hline BX Del......... & 1.09 & CWB: & II & AHB3 & 4 & 7,7 & $5^{\mathrm{a}}$ & $51,837-52,913$ & $35(18)$ & 1 \\
\hline 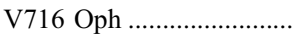 & 1.12 & CWB & II & AHB1 & 3 & 18,20 & 3 & $52,164-53,094$ & $62(13)$ & 2 \\
\hline 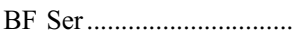 & 1.17 & CWB & II & AHB1 & 2 & 6,13 & 5 & $50,173-52,774$ & $65(31)$ & 3,4 \\
\hline 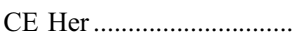 & 1.21 & CWB & II & AHB1 & 2 & $\ldots$ & $1^{\mathrm{b}}$ & $52,751-52,811$ & $150(12)$ & 4,5 \\
\hline 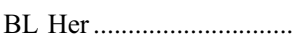 & 1.31 & CWB & II & AHB3 & 3 & 16,19 & $7^{\mathrm{a}}$ & $51,793-52,908$ & $135(29)$ & $4,6,7$ \\
\hline 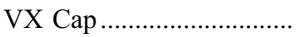 & 1.33 & CWB & II & AHB1 & 4 & 16,16 & $6^{\mathrm{a}}$ & $52,500-52,934$ & $86(10)$ & 2 \\
\hline 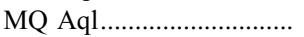 & 1.48 & CWB & $\ldots$ & (AHB1) & 2 & 10,12 & 8 & $51,800-52,822$ & $54(31)$ & 3 \\
\hline 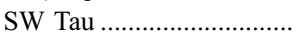 & 1.58 & CWB & II & AHB3 & 3 & 5,13 & $3^{\mathrm{a}}$ & $51,837-52,713$ & $214(36)$ & $3,6,7,8$ \\
\hline 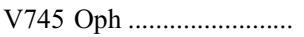 & 1.60 & CWB & II & AHB2 & 3 & 18,14 & 4 & $51,793-52,810$ & $65(25)$ & 2 \\
\hline 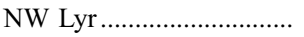 & 1.60 & CWB & II & (AHB2) & 2 & 8,8 & 12 & $51,793-52,898$ & $55(33)$ & 9 \\
\hline 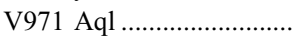 & 1.62 & CWB & II & AHB2 & 3 & 10,10 & 6 & $52,500-52,908$ & $70(24)$ & 2 \\
\hline 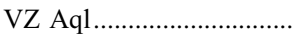 & 1.67 & CWB & II & AHB2 & 4 & 13,12 & 5 & $51,800-52,898$ & $50(17)$ & 2 \\
\hline 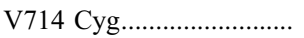 & 1.89 & CWB & II & $(\mathrm{AHB} 2:)^{\mathrm{c}}$ & 5 & 21,19 & 4 & $52,164-52,810$ & $73(50)$ & 3 \\
\hline 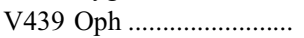 & 1.89 & CWB & II & AHB2 & 2 & 20,17 & 12 & $48,150-52,811$ & $100(39)$ & $3,10,11,12$ \\
\hline 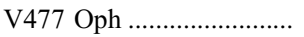 & 2.02 & CWB & II & AHB2 & 3 & 20,24 & 4 & $52,415-52,843$ & $102(23)$ & $1,2,13$ \\
\hline 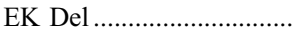 & 2.05 & CEP & II & AHB1 & 1 & 11,11 & 7 & $52,164-52,898$ & $70(17)$ & 2 \\
\hline 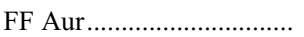 & 2.12 & DCEP & $\ldots$ & (AHB1) & 3 & 16,19 & 4 & $52,249-52,751$ & $100(23)$ & $1,3,4,14$ \\
\hline 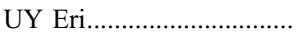 & 2.21 & CWB & II & AHB1 & 2 & 7,8 & $7^{\mathrm{d}}$ & $51,837-52,936$ & $78(37)$ & 4,15 \\
\hline 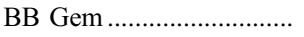 & 2.31 & DCEP & $\ldots$ & $\mathrm{C} \delta$ & 3 & 11,10 & 8 & $51,900-53,112$ & $98(54)$ & 3,6 \\
\hline 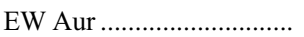 & 2.66 & DCEP & $\ldots$ & (AHB1) & 4 & 7,9 & 6 & $52,249-52,942$ & $140(34)$ & $1,4,14$ \\
\hline 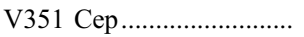 & 2.81 & CWB & $\ldots$ & $\mathrm{SA}$ & 2 & 10,14 & 10 & $51,837-52,885$ & $91(43)$ & 3,6 \\
\hline 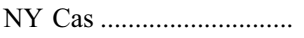 & 2.82 & DCEPS & $\ldots$ & $(\mathrm{SA})$ & 4 & 6,6 & 6 & $52,249-52,867$ & $159(26)$ & $1,3,9$ \\
\hline 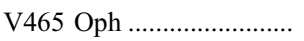 & 2.84 & CWB & II & $\mathrm{AHB} 3$ or $\mathrm{C} \delta^{\mathrm{e}}$ & 5 & 14,15 & 4 & $52,447-52,850$ & $64(22)$ & 2 \\
\hline 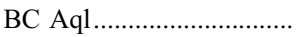 & 2.91 & CEP & $\ldots$ & $\mathrm{C} \delta$ & 2 & 15,17 & 9 & $52,456-52,898$ & $67(22)$ & 1 \\
\hline
\end{tabular}

a The zero point of our $V$ magnitudes was adjusted to produce agreement with earlier photometry.

${ }^{\mathrm{b}}$ For CE Her the zero points for both $V$ and $R$ were set by comparing the present photometry with that of Loomis et al. (1988). See text.

${ }^{c}$ We have classed V714 Cyg as AHB2 based on the bump on rising light, but it is unique among our sample in also having a bump on the falling branch of the light curve.

${ }^{\mathrm{d}}$ For UY Eri the zero point of the $V$ magnitudes transformed from Pel's (1976) Walraven photometry was adjusted. See text.

e The type of V465 Oph is uncertain. We consider it to be AHB3, given that it is a high- $Z$ star.

RefERenCES.-(1) Berdnikov 1987, 1992a, 1992b, 1992c, 1992d, 1992e, 1993; (2) Kwee \& Diethelm 1984; (3) Henden 1980, 1996; (4) Harris 1980; (5) Loomis et al. 1988; (6) Szabados 1977; (7) Moffett \& Barnes 1984; (8) Barnes et al. 1997; (9) Schmidt \& Reiswig 1993; (10) Diethelm \& Tammann 1982; (11) Sturch 1966; (12) Harris \& Wallerstein 1984; (13) Schmidt \& Seth 1996; (14) Schmidt et al. 1995; (15) Pel 1976 (Walraven photometry transformed to the Johnson system using $V_{\mathrm{J}}=6.874-2.5\left[V_{W}-0.065\left(V_{W}-B_{W}\right)\right]$ from the same reference).

with those from earlier photometry (references in col. [11]). When significant zero-point shifts were apparent, we adjusted our $V$ magnitudes to match the earlier photometry before combining all of the data for analysis. Such cases are identified by footnotes to the table. Unfortunately, no such check is possible for the $R$ magnitudes. For UY Eri the photometry of Harris (1980) matched our photometry in zero point, while the $V$ magnitudes transformed from the Walraven photometry of Pel (1976) did not. Thus, we adjusted the Pel data to match the other two. For CE Her, we had data from only one photometric night. In that case the magnitudes of the variable were compared with those from Loomis et al. (1988), and the zero points for both $V$ and $R$ were set to achieve agreement.

TABLE 2

Рнотометric Data

\begin{tabular}{|c|c|c|c|c|}
\hline Star & HJD $-2,400,000$ & $V$ & $R$ & $V-R$ \\
\hline BX Del... & $51,837.700$ & 12.139 & 11.805 & 0.334 \\
\hline 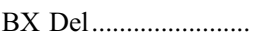 & $52,540.751$ & 12.130 & 11.807 & 0.320 \\
\hline 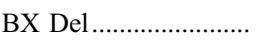 & $52,832.886$ & 12.509 & 12.128 & 0.377 \\
\hline 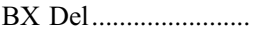 & $52,851.844$ & 12.070 & 11.757 & 0.310 \\
\hline
\end{tabular}

Note.-Table 2 is presented in its entirety in the electronic edition of the Astronomical Journal. A portion is shown here for guidance regarding its form and content.
In column (9) we list the range of Julian Dates of our observations. In our analysis we have incorporated $V$ photometry from the literature for most of the stars in addition to our new observations. In column (10) the first number indicates the total number of light-curve points available, while the number in parentheses is the number of new points presented here. Finally, column (11) provides references to the sources of the earlier photometry.

In Table 2 we list the new observations. The $V$ magnitudes in this table have not been adjusted as described above. The first author will provide complete tabulations of all of the data for each star, including those from the literature, upon request.

\section{PROPERTIES OF THE LIGHT CURVES}

In Table 3 we list various parameters describing the light curves. Column (1) lists the star names, while Column (2) gives the adopted periods, most of which are newly determined. The footnotes indicate what data were used in determining these periods. Column (3) gives upper limits on the uncertainty of the periods in units of the last cited digit of the period. In column (4) we list $\Delta P$, which is the difference between the period in column (2) and the period from the GCVS. It is also expressed in units of the last digit of the period. Since our periods refer to a later epoch than those from the GCVS, positive values of $\Delta P$ correspond to increased periods. In column (5) we list the epoch of maximum 
TABLE 3

The Light-Curve Parameters

\begin{tabular}{|c|c|c|c|c|c|c|c|c|c|c|}
\hline $\begin{array}{l}\text { Star } \\
(1)\end{array}$ & $\begin{array}{c}\text { Period } \\
\text { (days) } \\
(2)\end{array}$ & $\begin{array}{l}\sigma_{P} \\
(3)\end{array}$ & $\begin{array}{l}\Delta P \\
(4)\end{array}$ & $\begin{array}{c}\mathrm{HJD}_{\max }-2,400,000 \\
(5)\end{array}$ & $\begin{array}{c}\langle V\rangle \\
(\mathrm{mag}) \\
(6)\end{array}$ & $\begin{array}{c}\langle R\rangle \\
(\mathrm{mag}) \\
(7)\end{array}$ & $\begin{array}{c}\Delta V \\
(\mathrm{mag}) \\
(8)\end{array}$ & $\begin{array}{c}\Delta R \\
(\mathrm{mag}) \\
(9)\end{array}$ & $\begin{array}{l}\phi_{\min } \\
(10)\end{array}$ & $\begin{array}{l}\text { Notes } \\
(11)\end{array}$ \\
\hline BX Del................................. & 1.09179 & 1 & 1 & $52,886.58$ & 12.18 & 11.8 & 0.75 & 0.6 & 0.75 & a \\
\hline 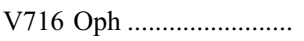 & 1.115920 & 2 & 4 & $52,872.42$ & 12.03 & $\ldots$ & 1.40 & $\ldots$ & 0.87 & a \\
\hline 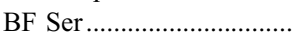 & 1.1654394 & 50 & 0 & $52,755.61$ & 11.97 & 11.76 & 1.50 & $\ldots$ & 0.91 & $\mathrm{~b}$ \\
\hline 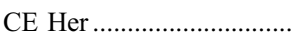 & 1.2094357 & 30 & 0 & $52,767.62$ & 12.30 & 12.06 & 1.34 & 1.10 & 0.91 & $\mathrm{~b}$ \\
\hline 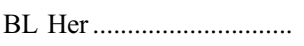 & 1.307442 & 2 & -8 & $52,780.65$ & 10.17 & 9.85 & 0.87 & 0.66 & 0.78 & a \\
\hline 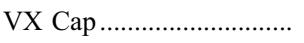 & 1.327546 & 5 & -12 & $52,865.70$ & 14.90 & 14.61 & 1.32 & 1.24 & 0.89 & $\mathrm{c}$ \\
\hline 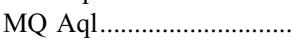 & 1.48078 & 2 & 4 & $52,811.66$ & 13.77 & 13.31 & 1.37 & 0.95 & 0.82 & a, d \\
\hline 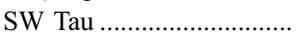 & 1.583558 & 5 & -26 & $52,665.04$ & 9.70 & 9.26 & 0.87 & 0.84 & 0.62 & a, e \\
\hline 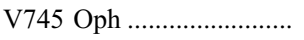 & 1.5962 & 1 & 11 & $52,769.73$ & 13.17 & 12.86 & 0.95 & 0.80 & 0.69 & f \\
\hline 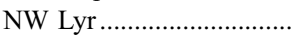 & 1.6011823 & 100 & 0 & $52,885.66$ & 12.47 & 12.13 & 1.08 & 0.86 & 0.70 & $\mathrm{~b}$ \\
\hline 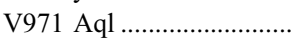 & 1.624525 & 5 & 29 & $52,867.56$ & 11.97 & 11.56 & 0.85 & 0.59 & 0.70 & a \\
\hline 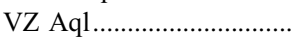 & 1.66827 & 1 & 3 & $52,885.62$ & 13.52 & 13.03 & 1.00 & 0.72 & 0.74 & a \\
\hline 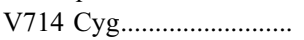 & 1.88744 & 3 & 7 & $52,425.73$ & 14.10 & 13.73 & 1.32 & 1.08 & 0.74 & a \\
\hline 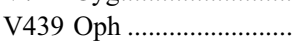 & 1.89301 & 1 & 3 & $52,801.63$ & 12.16 & 11.65 & 0.71 & 0.53 & 0.69 & a \\
\hline 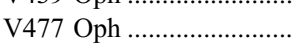 & 2.01569 & 1 & 2 & $52,488.77$ & 13.83 & 13.43 & 0.85 & 0.66 & 0.71 & a \\
\hline EK Del ................................. & 2.047232 & 3 & 527 & $52,872.59$ & 12.33 & 12.01 & 0.91 & 0.70 & 0.80 & a \\
\hline 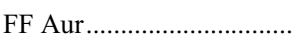 & 2.12055 & 1 & 2 & $52,618.62$ & 13.72 & 13.14 & 1.12 & 0.92 & 0.89 & a \\
\hline 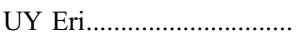 & 2.213280 & 5 & 45 & $51,942.59$ & 11.25 & 10.94 & 0.70 & 0.61 & 0.79 & a \\
\hline BB Gem & 2.30821 & 1 & 0 & $51,942.67$ & 11.44 & 10.94 & 1.11 & 0.89 & 0.83 & $\mathrm{~g}$ \\
\hline EW Aur & 2.65954 & 1 & -2 & $52,705.70$ & 13.53 & 12.87 & 0.87 & 0.68 & 0.83 & a \\
\hline 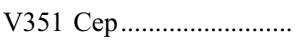 & 2.8065 & 1 & 6 & $52,855.75$ & 9.41 & 8.85 & 0.36 & 0.28 & 0.57 & a \\
\hline 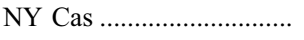 & 2.82315 & 3 & 6 & $52,521.98$ & 13.33 & 12.81 & 0.46 & 0.40 & 0.56 & a \\
\hline 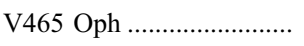 & 2.84394 & 2 & -65 & $52,820.61$ & 13.42 & 12.84 & 1.10 & 0.85 & 0.88 & a \\
\hline 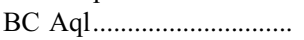 & 2.90526 & 3 & 13 & $52,886.69$ & 13.13 & 12.64 & 1.01 & 0.60 & 0.91 & a \\
\hline
\end{tabular}

\footnotetext{
${ }^{\mathrm{a}}$ The new period and light-curve parameters were determined from all of the available data.

$\mathrm{b}$ The new period is not significantly different from the GCVS period, therefore the latter was used. The light-curve parameters were determined from all of the data.

${ }^{c}$ Our photometry of VX Cap was limited to phases from about maximum light through minimum. The light-curve point nearest maximum and that nearest minimum were both too bright compared with the photometry of Kwee \& Diethelm (1984), therefore we have corrected our $V$ magnitudes to produce agreement. Without this correction the period derived would be $1.327552 \pm 0.000004$ days. While this is closer to the GCVS period, the fit of the photometry is poorer. Further data are needed to resolve this ambiguity.

d Because of the large scatter, the period for MQ Aql is poorly defined. The cited value was derived by minimizing scatter during rising light.

e For SW Tau the light-curve parameters, other than the period, were determined from the present photometry only.

${ }^{\mathrm{f}}$ The period listed for V745 Oph is based on the present data. The Kwee \& Diethelm photometry required a period that is smaller by 0.0007 days. Only the present data were used for deriving the light-curve parameters.

${ }^{\mathrm{g}}$ The period for BB Gem was determined from the present photometry and that of Henden (1980). Only the present photometry was used to derive the other photometric parameters.
}

based on the most recent photometry. Sampling and scatter can make the time of maximum uncertain. To address this in a consistent fashion, we have estimated the time of maximum by linearly extrapolating the rising and declining branches of the light curve to the point where they meet. There are stars with rather flat maxima or double maxima, which makes the selection of the time of maximum ambiguous. The reader can judge how this was handled by examining the light curves in Figure 1. In columns (6)(9) we list the intensity mean magnitudes and the amplitudes in $V$ and $R$. Finally, we have provided the phase of minimum light in column (10). This was determined by linear extrapolation, as was the maximum. Some of the stars have poorly defined minima, and again the reliability of the phases of minimum can be assessed by looking at the light curves themselves.

Figure 1 presents plots of the light curves including both our data and that from the references in column (11) of Table 1. The ephemerides in Table 3 were used for phasing the data for all but one of the stars. For V745 Oph no period could be found that fit both our data and that of Kwee \& Diethelm (1984). Hence, the two sets of observations were each phased using its own ephemeris. For some stars different symbols distinguish particular subsets of the data. The subsets that are associated with various symbols are defined below in $\S$ 4.2.1.
We have fitted Fourier series to all of our light curves. The order of the adopted fit for each star was selected to avoid overfitting of the data while minimizing the scatter. The Fourier parameters (see Simon \& Lee 1981 for definitions of them) are listed in Table 4. The errors were calculated using the scheme of Petersen (1986). Larger errors are associated with light curves with greater scatter.

To measure the scatter in the light curves we have calculated the standard deviations of individual data points about the fitted curve. When the light curve is very asymmetric, the Fourier fit is generally poor from before minimum until after maximum light. In those cases, we have removed points at which the fit was poor and calculated the scatter with the remainder of the data set. The standard deviations are listed in the last column of Table 4.

\section{DISCUSSION}

\subsection{Form of the Light Curves}

In Figure 2 we have plotted the Fourier parameters against period for stars from the present sample, as well as for stars from Moffett \& Barnes (1985), Petersen \& Diethelm (1986), Antonello \& Poretti (1986), Antonello et al. (1990), and Mantegazza \& 

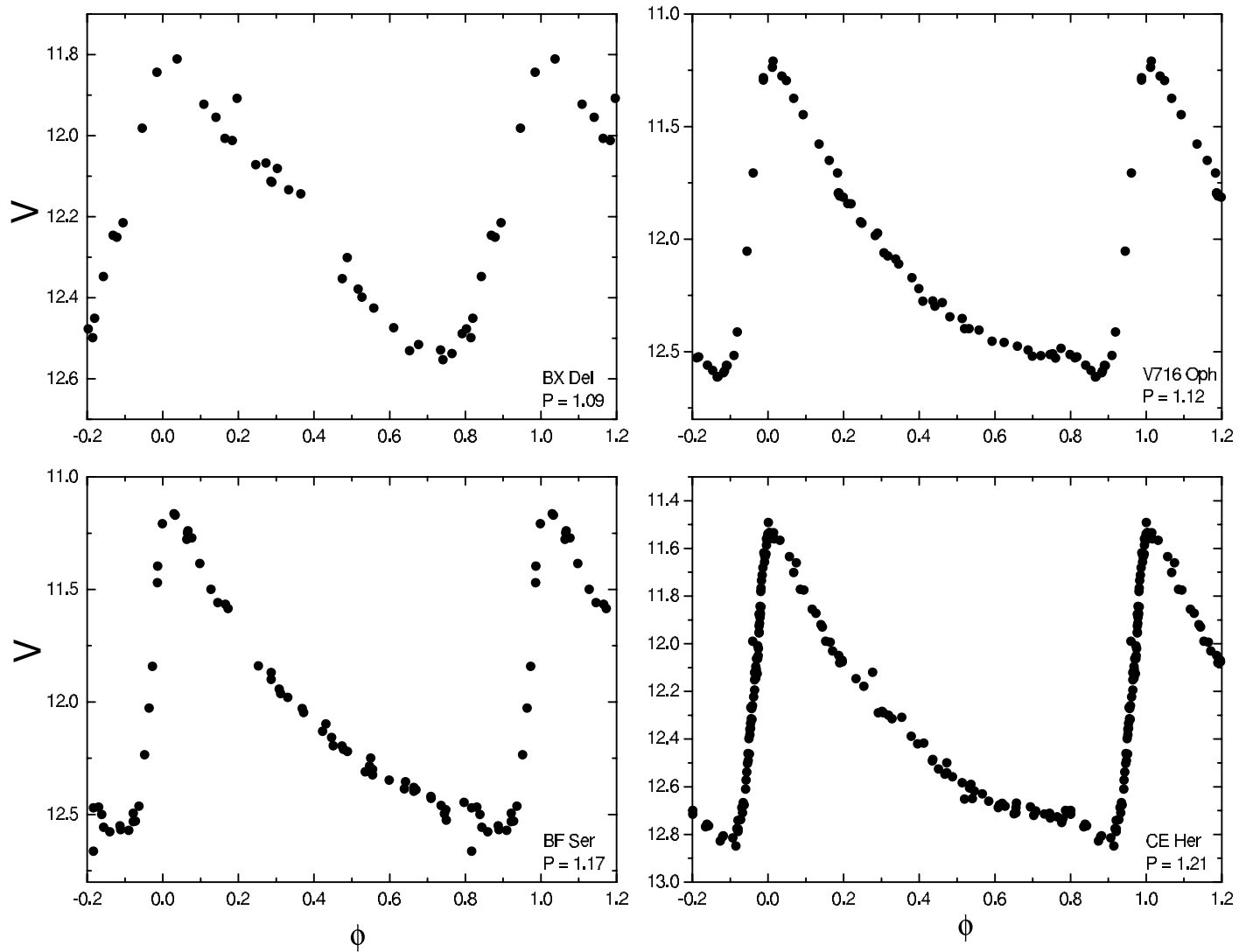

FIG. $1 a$
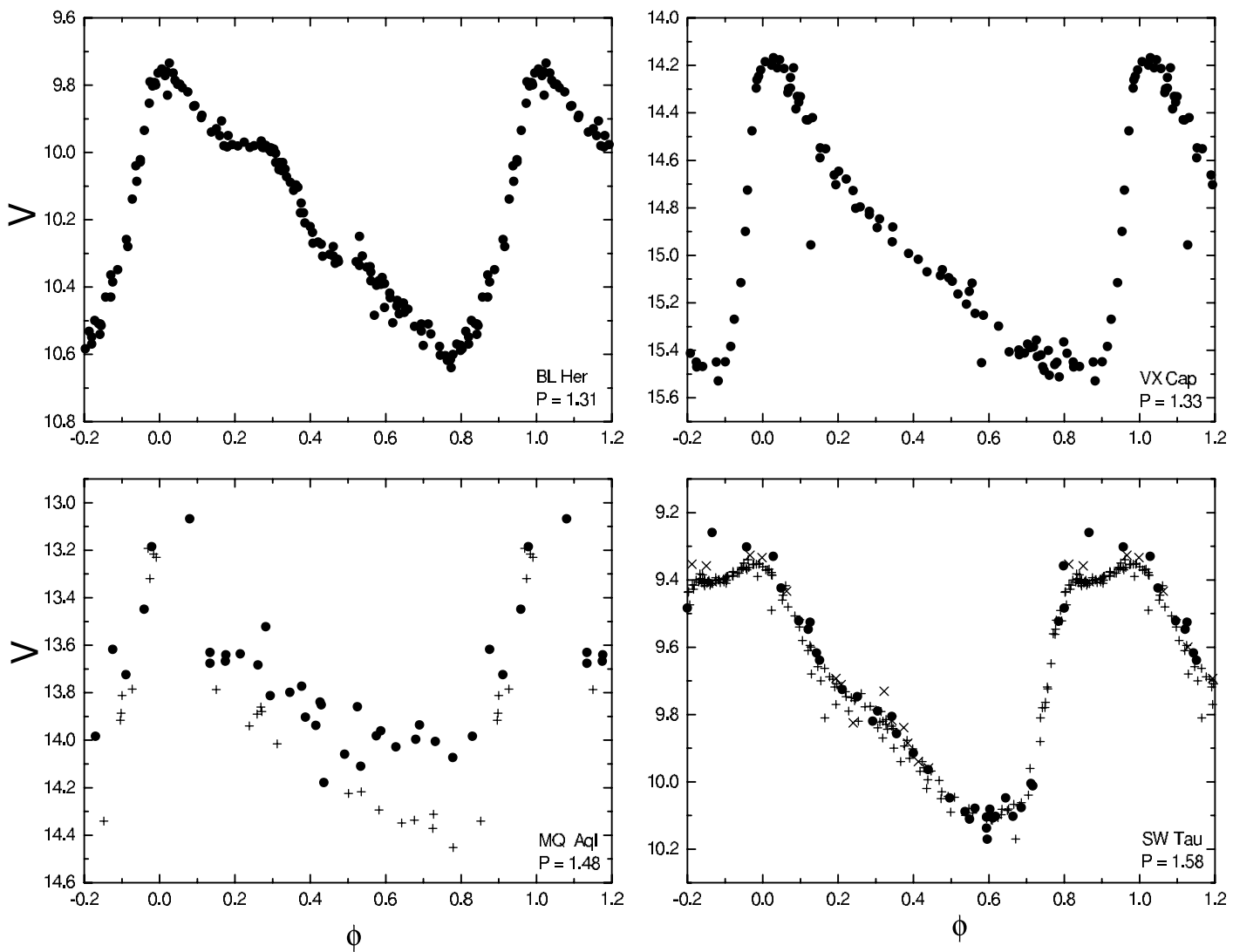

FIG. $1 b$

Fig. 1.-The $V$-magnitude light curves for the stars listed in Table 1. In some cases different symbols denote different subsets of data as described in the text. 

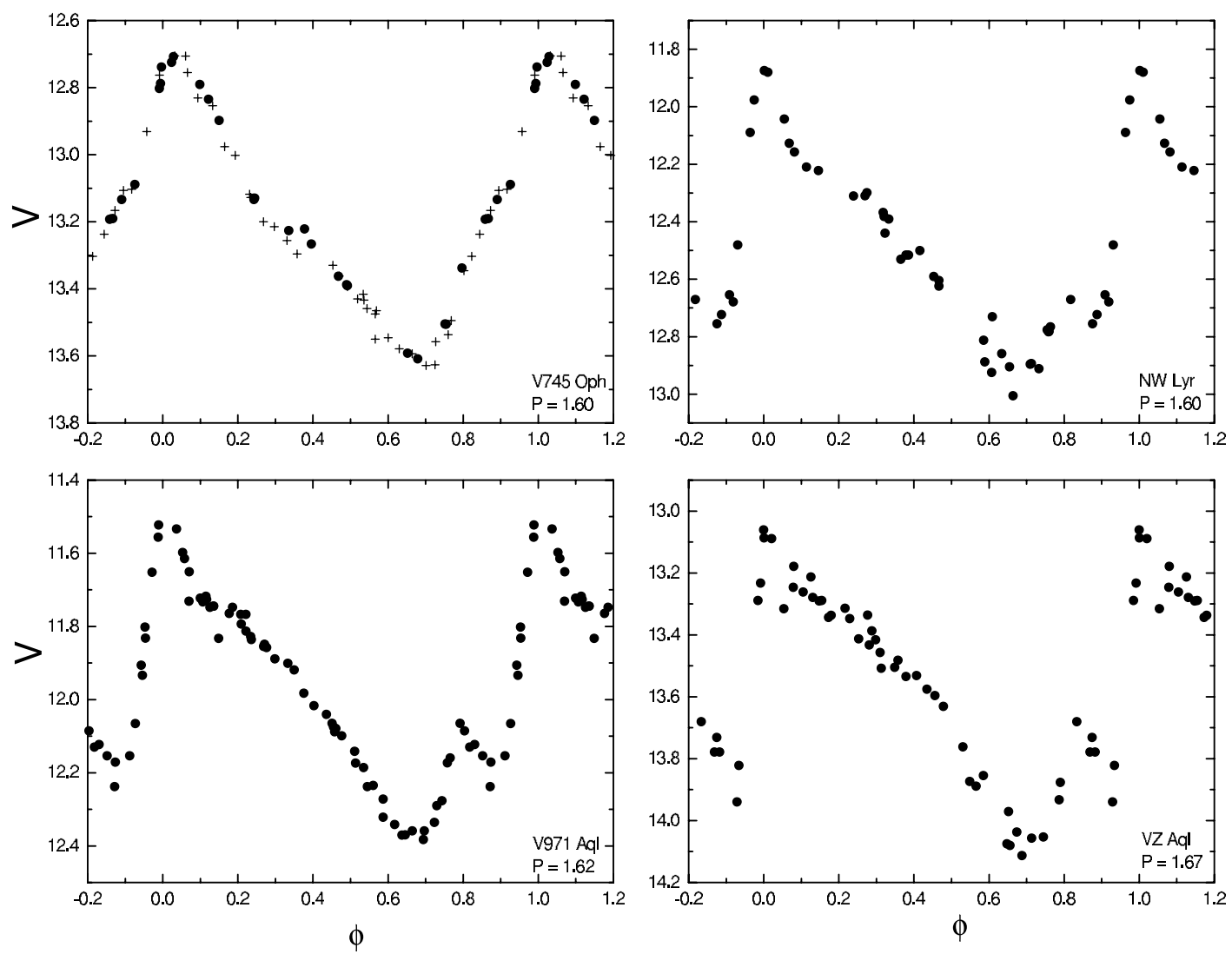

FIG. $1 c$
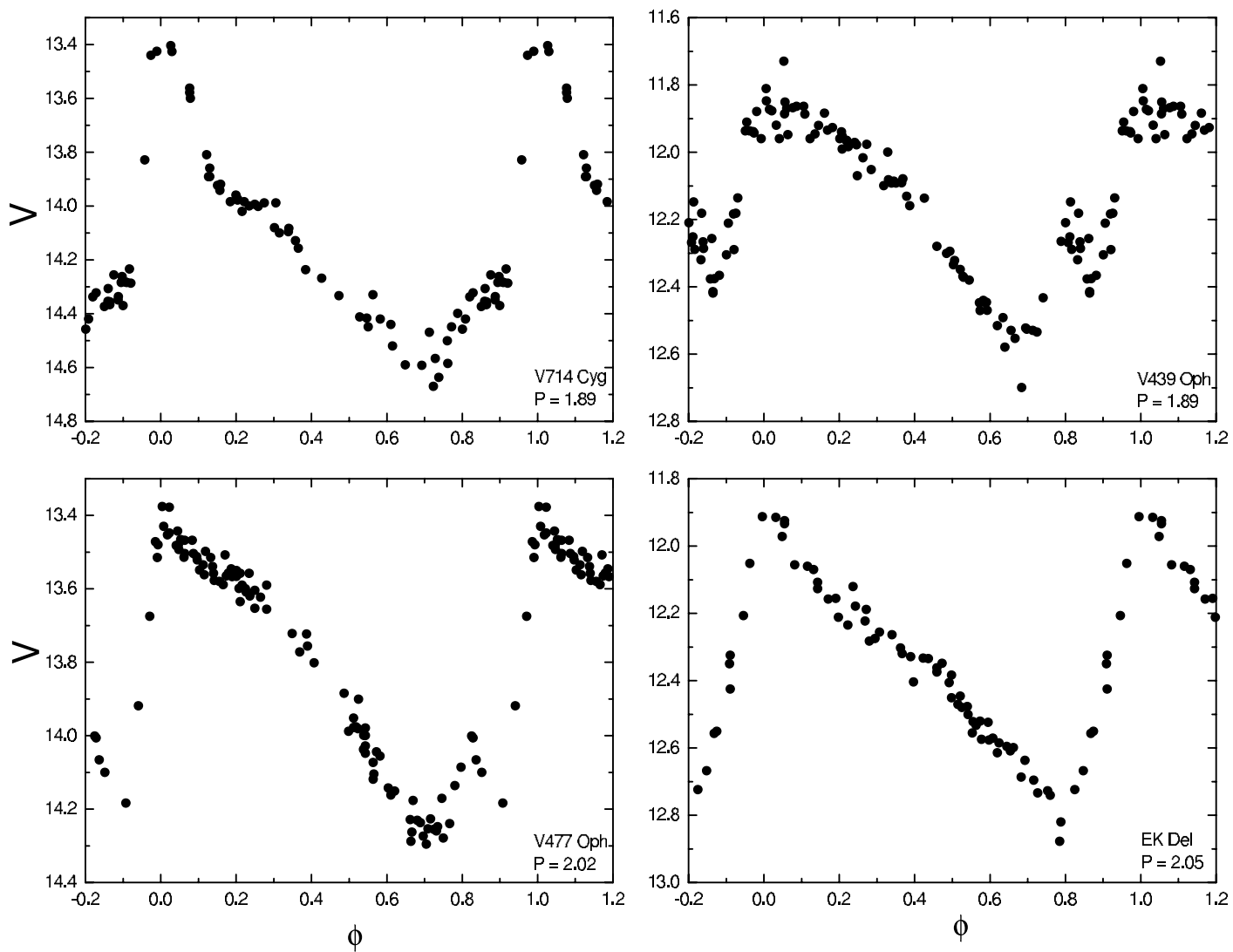

FIG. $1 d$ 

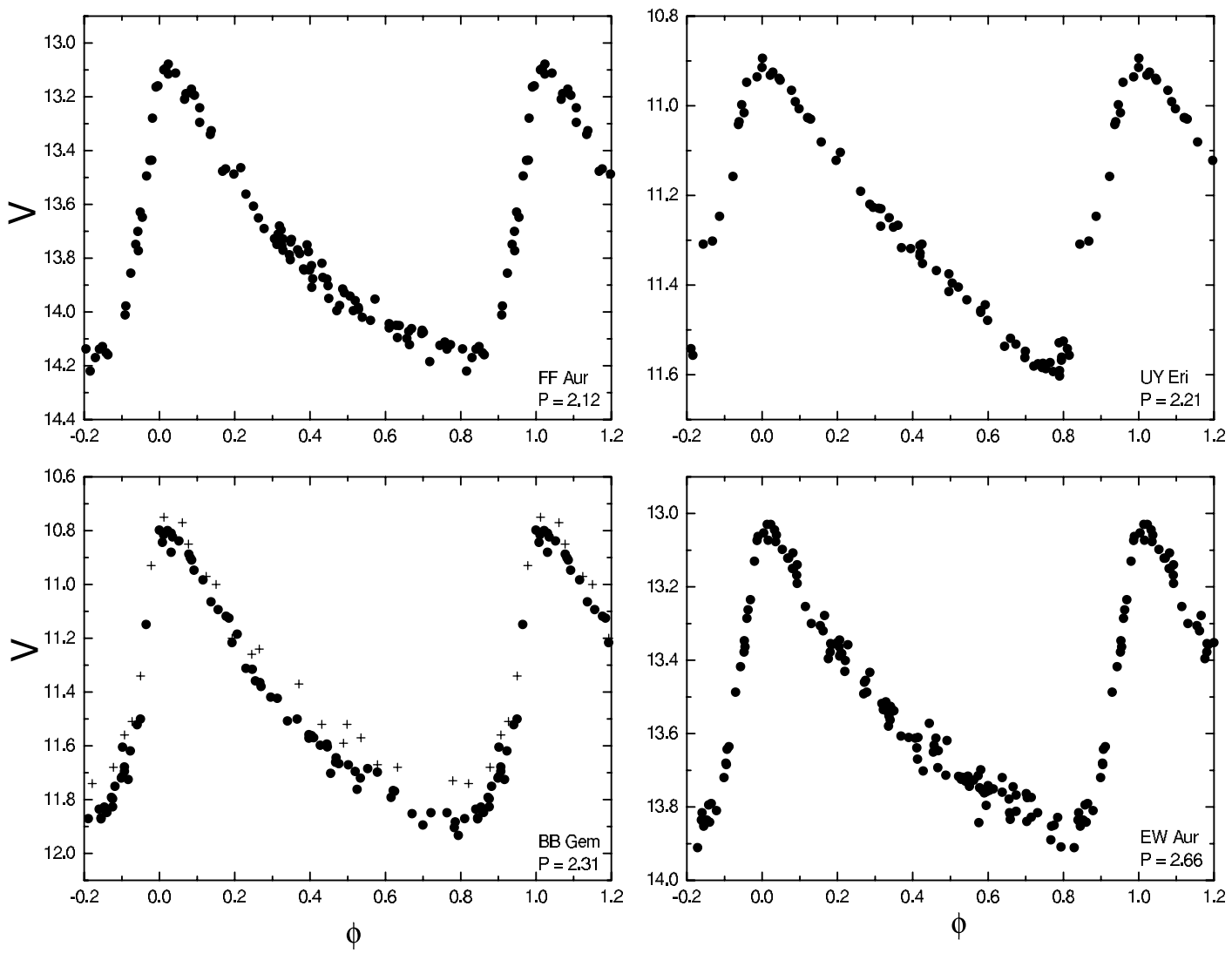

FIG. 1e
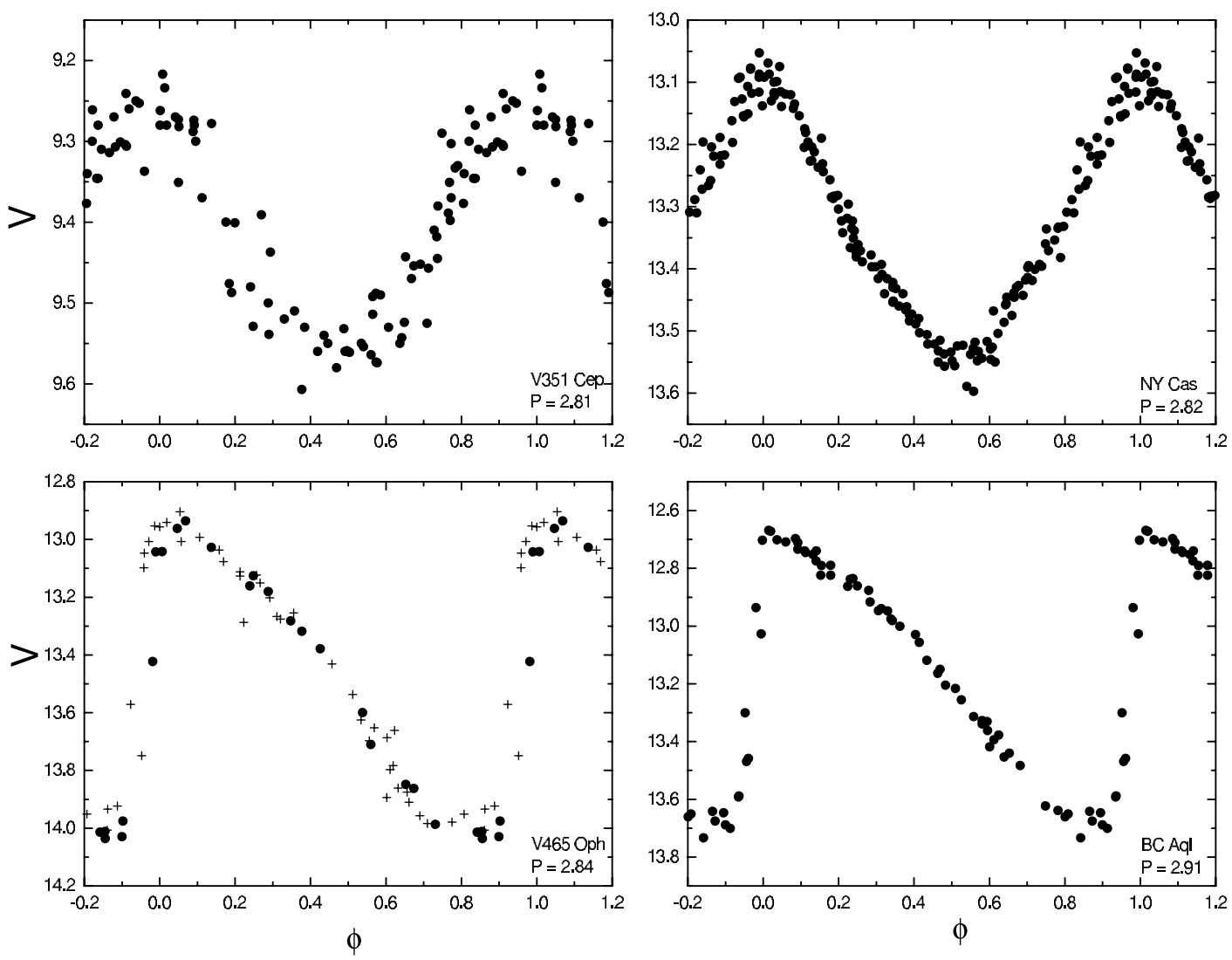

FIG. $1 f$ 
TABLE 4

The Fourier Parameters

\begin{tabular}{|c|c|c|c|c|c|c|c|c|c|c|c|c|c|}
\hline $\begin{array}{c}\text { Star } \\
\text { (1) }\end{array}$ & $\begin{array}{l}R_{21} \\
(2)\end{array}$ & $\begin{array}{c}\sigma \\
(3)\end{array}$ & $\begin{array}{l}R_{31} \\
(4)\end{array}$ & $\begin{array}{c}\sigma \\
(5)\end{array}$ & $\begin{array}{l}R_{41} \\
\text { (6) }\end{array}$ & $\begin{array}{c}\sigma \\
(7)\end{array}$ & $\begin{array}{c}\phi_{21} \\
(8)\end{array}$ & $\begin{array}{c}\sigma \\
(9)\end{array}$ & $\begin{array}{c}\phi_{31} \\
(10)\end{array}$ & $\begin{array}{c}\sigma \\
(11)\end{array}$ & $\begin{array}{c}\phi_{41} \\
(12)\end{array}$ & $\begin{array}{c}\sigma \\
(13)\end{array}$ & $\begin{array}{c}\sigma_{V} \\
(14)\end{array}$ \\
\hline 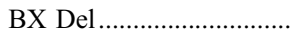 & 0.32 & 0.03 & 0.13 & 0.02 & 0.04 & 0.02 & 4.77 & 0.09 & 2.64 & 0.19 & 1.02 & 0.57 & 0.022 \\
\hline 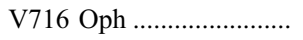 & 0.48 & 0.02 & 0.32 & 0.02 & 0.21 & 0.01 & 4.00 & 0.04 & 1.89 & 0.06 & 6.07 & 0.09 & 0.025 \\
\hline 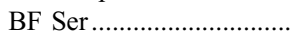 & 0.51 & 0.02 & 0.32 & 0.02 & 0.21 & 0.02 & 4.14 & 0.05 & 2.17 & 0.07 & 0.22 & 0.10 & 0.029 \\
\hline 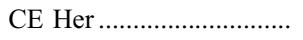 & 0.49 & 0.01 & 0.38 & 0.01 & 0.27 & 0.01 & 4.05 & 0.04 & 1.94 & 0.05 & 6.13 & 0.07 & 0.024 \\
\hline 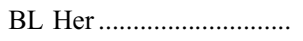 & 0.35 & 0.01 & 0.24 & 0.01 & 0.11 & 0.01 & 4.72 & 0.03 & 3.31 & 0.05 & 0.58 & 0.10 & 0.026 \\
\hline VX Cap & 0.52 & 0.02 & 0.30 & 0.02 & 0.21 & 0.02 & 4.38 & 0.05 & 2.35 & 0.08 & 0.41 & 0.11 & 0.040 \\
\hline MQ Aql............................. & 0.52 & 0.09 & 0.37 & 0.08 & $\ldots$ & $\ldots$ & 4.17 & 0.22 & 1.78 & 0.32 & $\ldots$ & $\ldots$ & 0.159 \\
\hline 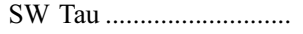 & 0.35 & 0.01 & 0.08 & 0.01 & 0.12 & 0.01 & 4.52 & 0.03 & 3.53 & 0.11 & 1.70 & 0.08 & 0.031 \\
\hline 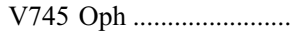 & 0.32 & 0.02 & 0.09 & 0.02 & 0.11 & 0.02 & 4.34 & 0.08 & 0.63 & 0.25 & 4.10 & 0.21 & 0.027 \\
\hline 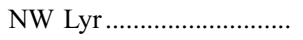 & 0.24 & 0.02 & 0.18 & 0.02 & 0.25 & 0.02 & 4.76 & 0.10 & 1.88 & 0.14 & 6.13 & 0.13 & 0.045 \\
\hline V971 Aq1 ................................ & 0.22 & 0.01 & 0.18 & 0.01 & 0.26 & 0.01 & 4.74 & 0.07 & 1.60 & 0.08 & 6.08 & 0.07 & 0.025 \\
\hline 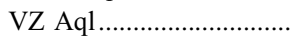 & 0.23 & 0.03 & 0.15 & 0.03 & 0.18 & 0.03 & 5.15 & 0.14 & 1.57 & 0.21 & 0.09 & 0.19 & 0.054 \\
\hline 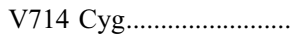 & 0.41 & 0.02 & 0.25 & 0.02 & 0.24 & 0.02 & 4.30 & 0.07 & 1.88 & 0.10 & 5.29 & 0.12 & 0.041 \\
\hline 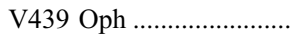 & 0.20 & 0.03 & 0.17 & 0.03 & 0.15 & 0.03 & 4.96 & 0.14 & 1.67 & 0.16 & 0.36 & 0.19 & 0.040 \\
\hline 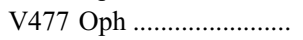 & 0.24 & 0.01 & 0.16 & 0.01 & 0.19 & 0.01 & 5.02 & 0.06 & 2.39 & 0.09 & 0.81 & 0.08 & 0.033 \\
\hline EK Del ................................. & 0.49 & 0.02 & 0.23 & 0.02 & 0.12 & 0.02 & 4.85 & 0.05 & 3.11 & 0.10 & 1.58 & 0.18 & 0.035 \\
\hline FF Aur................................... & 0.47 & 0.02 & 0.27 & 0.01 & 0.16 & 0.01 & 4.10 & 0.04 & 2.05 & 0.07 & 6.22 & 0.11 & 0.036 \\
\hline 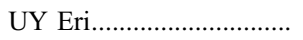 & 0.40 & 0.01 & 0.16 & 0.01 & 0.07 & 0.01 & 4.48 & 0.04 & 2.95 & 0.09 & 1.07 & 0.20 & 0.023 \\
\hline 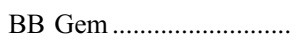 & 0.48 & 0.02 & 0.21 & 0.02 & 0.13 & 0.02 & 4.12 & 0.05 & 2.08 & 0.09 & 6.11 & 0.14 & 0.035 \\
\hline 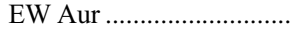 & 0.48 & 0.01 & 0.28 & 0.01 & 0.16 & 0.01 & 4.17 & 0.03 & 2.20 & 0.06 & 0.13 & 0.09 & 0.030 \\
\hline 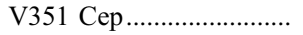 & 0.07 & 0.04 & 0.06 & 0.04 & 0.08 & 0.04 & 3.22 & 0.55 & 4.20 & 0.73 & 0.80 & 0.52 & 0.040 \\
\hline 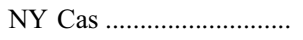 & 0.10 & 0.01 & 0.09 & 0.01 & 0.02 & 0.01 & 3.43 & 0.12 & 6.08 & 0.13 & 4.91 & 0.54 & 0.024 \\
\hline 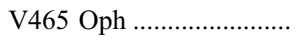 & 0.38 & 0.04 & 0.20 & 0.04 & 0.14 & 0.04 & 5.00 & 0.13 & 3.28 & 0.24 & 1.80 & 0.33 & 0.047 \\
\hline 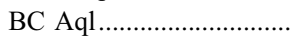 & 0.38 & 0.03 & 0.23 & 0.03 & 0.16 & 0.03 & 4.89 & 0.08 & 3.29 & 0.13 & 1.58 & 0.20 & 0.030 \\
\hline
\end{tabular}
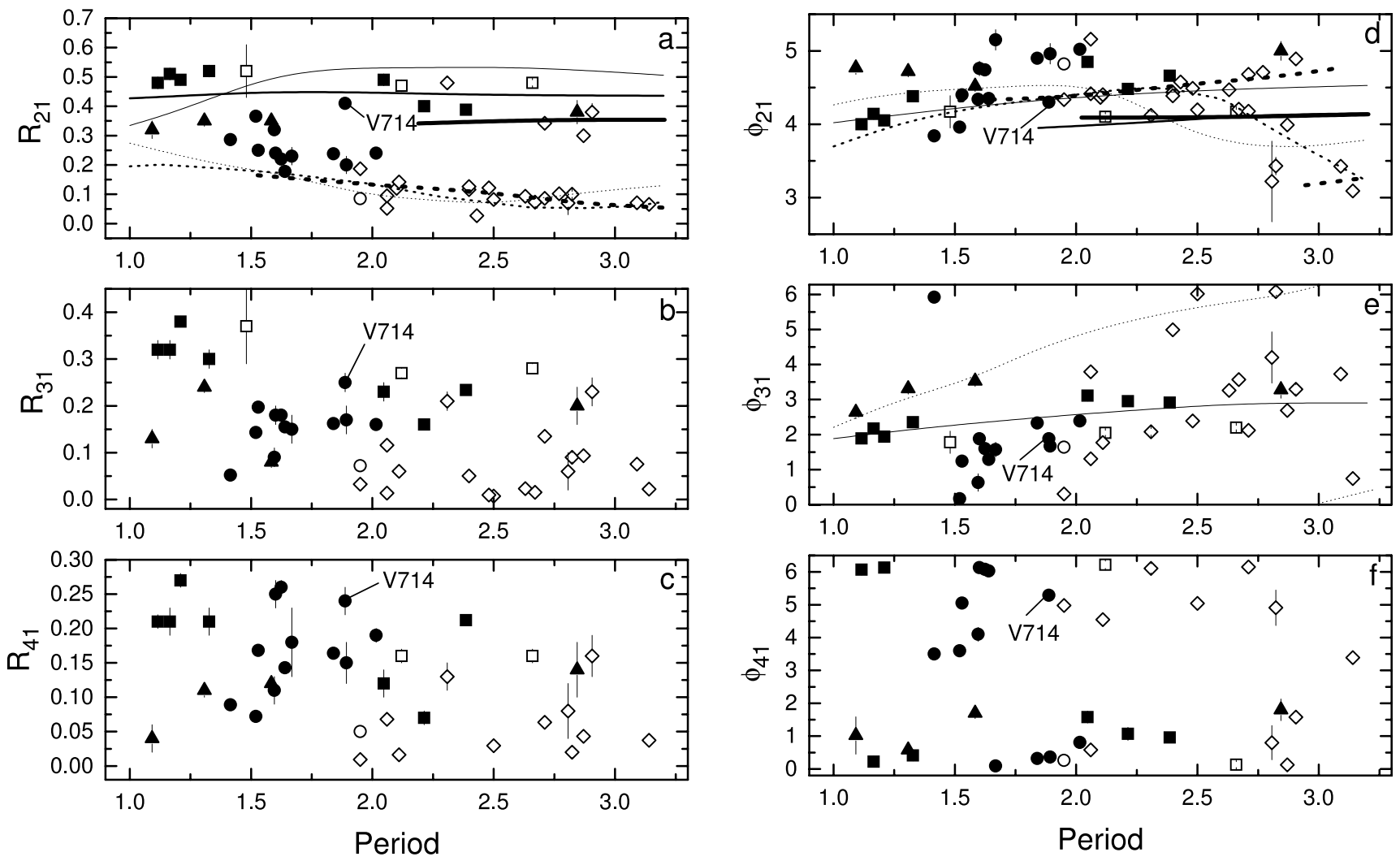

Fig. 2.-Fourier parameters plotted against period. Open symbols represent low- $Z$ stars, and filled symbols represent high- $Z$ stars. For stars from this study and from Petersen \& Diethelm (1986) the shape of the symbols denotes the form of the light curve as follows: squares, AHB1; circles, AHB2; triangles, AHB3; and diamonds, $\mathrm{C} \delta$ and SA. Stars from the other sources are presumed to be classical Cepheids and are plotted as diamonds. Error bars are shown for stars from the present study when they are larger than the plotted symbol. V714 Cyg is discussed in the text and is marked for easy reference. Mean curves are plotted for classical Cepheids from the Galaxy (thick lines), the Large Magellanic Cloud (intermediate-weight lines), and the Small Magellanic Cloud (thin lines). The relations for fundamental pulsators are plotted as solid lines, and those for first-overtone pulsators are plotted as dotted lines. 
Poretti (1992). Various symbols distinguish high- and low- $Z$ stars and stars with various types of light curves, as described in the figure legend.

For classical Cepheids in the Galaxy, the LMC, and the SMC, Poretti \& Pardo (1997, Figure 4), Alcock et al. (1999, Figure 4), Udalski et al. (1999a, Figure 3), and Udalski et al. (1999b, Figure 3 ) presented similar plots for $R_{21}, \phi_{21}$, and $\phi_{31}$ (the SMC only). In these studies the pulsation modes of the variables have been identified by reference to the $\log P-V$ diagram and by Fourier decomposition of double-mode stars. The latter has also been useful in providing values of $R_{21}$ for the first overtone at shorter periods than are found among single-mode stars in the Galaxy. We have drawn mean relations through the sequences in those diagrams and plotted them in Figures $2 a, 2 d$, and $2 e$. It should be noted that the first-overtone sequences for $\phi_{21}$ are rather uncertain in the region where they cross the fundamental sequences. Our interpretation is that the Galaxy is double valued in that region, but we have drawn continuous sequences for the SMC and the LMC. The reader is advised to look at the original diagrams to gain a sense of the validity of our interpretation.

In Figure $2 a$, the $R_{21}$ diagram, the fundamental and overtone curves are well separated, and most of the low- $Z$ stars appear to be overtone pulsators. This is consistent with the discussion of Mantegazza \& Porretti (1992, and earlier papers by this same group) regarding the short-period stars with small values of $R_{21}$. The location of the low- $Z$ stars in Figure $2 d$, the $\phi_{21}$ diagram, is reasonably consistent with the $R_{21}$ diagram where the two modes are significantly separated. However, in the $\phi_{31}$ diagram the majority of the low- $Z$ stars cluster around the fundamental curve. The significance of this is unclear given the differences between the relations in Figure $2 d$ among the three stellar systems and the fact that we have mean sequences for the SMC only. We can speculate that at the metallicities of the Galactic classical Cepheids the overtone curve is shifted to the right by about 1.5 days. If this is the case, most of the low- $Z$ stars would fall reasonably close to the curve, considering the amount of scatter visible in Figure 3 of Udalski et al. (1999a). We conclude that only $R_{21}$ is useful at this point for inferring the pulsation modes of classical Cepheids.

In comparing the location of the type II Cepheids with the mean sequences, we note that the AHB2 stars (circles) fall fairly close to the overtone sequences in Figure $2 a$. This may suggest that they are overtone pulsators. Similarly, there is a suggestion in the diagram that the ABH1 stars (squares) are fundamental pulsators. We must treat these conclusions with caution, since we do not know how applicable the behavior of classical Cepheids is to type II Cepheids.

Smith et al. (1992) showed that the pulsation mode of SMC Cepheids was related to amplitude in the sense that all of the overtone pulsators they observed had $B$ amplitudes smaller than 1.05 mag. This corresponds to about $0.70 \mathrm{mag}$ in $V$. In our sample, only two stars, V351 Cep and NY Cas, have amplitudes less than this amount. They also fall along the overtone curves in Figure $2 a$. This suggests that the remainder of the stars in our sample are fundamental pulsators. Again, this is subject to the caveat that we are uncertain whether the amplitudes of the type II Cepheids are related to pulsation mode in the same way as the classical Cepheids. A definitive decision regarding the pulsation modes of the type II Cepheids must await further investigation of these stars in other stellar systems.

The AHB2 stars (circles) occupy the period range from 1.4 to 2 days, while the AHB1 (squares) and AHB3 stars (triangles) largely fall outside that range. This limits the usefulness of a comparison of the three types of light curves in Figure 2. How-
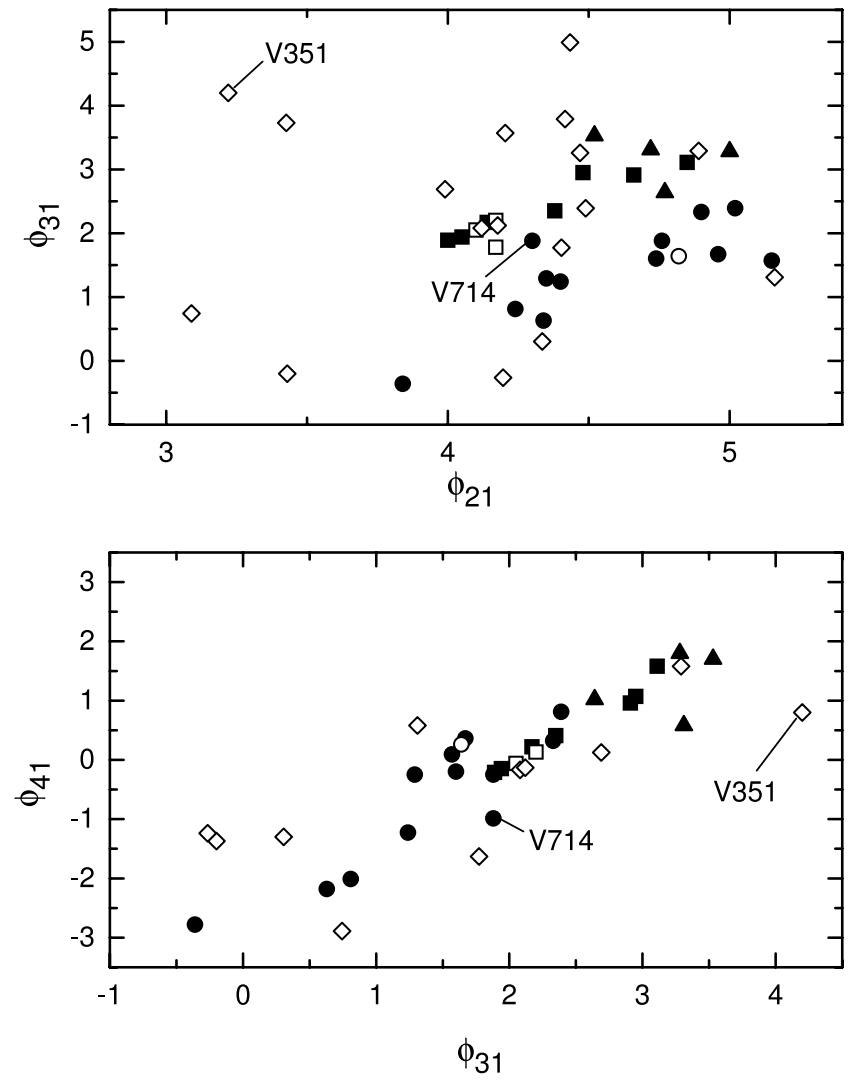

FIG. 3.-Fourier phase parameters plotted against each other. The symbols have the same meaning as in Fig. 2. The ranges for $\phi_{31}$ and $\phi_{41}$ have been extended to negative values to make the sequences of points more obvious. V351 Cep and V714 Cyg are mentioned in the text and are identified for easy reference.

ever, we note that the AHB1 stars have generally larger values of $R_{21}$ and $R_{31}$ than the other two types. This is not surprising given that their light curves are very asymmetric and require higher order Fourier terms.

In Figures $2 d-2 f$ it can be seen that of the phase differences, only $\phi_{31}$ shows a reasonable separation among the three types of light curves. However, in that diagram the three types form sequences that are sufficiently well separated to be useful.

Simon (1986) demonstrated that plots of the Fourier phase differences against each other were useful in revealing mode resonances, $P_{2} / P_{0} \approx 0.5$ in particular. We show two such plots in Figure 3, $\phi_{31}$ versus $\phi_{21}$ and $\phi_{41}$ versus $\phi_{31}$. The plot of $\phi_{41}$ versus $\phi_{21}$ is not shown since it is very similar to $\phi_{31}$ versus $\phi_{21}$. The appearance of the diagrams with regard to the high- $Z$ stars is very similar to that shown by Simon. In the top panel the AHB1 (squares) and AHB3 (triangles) stars form one sequence, while the $\mathrm{ABH} 2$ (circles) stars follow another sequence below it. In the bottom panel, we see that all of the high- $Z$ stars follow a single sequence.

Of the period diagrams in Figure 2, only $\phi_{31}$ versus period is useful for separating the three types of light curves. Alternatively, the combination of the $R_{21}$ versus period diagram with the $\phi_{31}$ versus $\phi_{21}$ diagram can be used to distinguish among them.

The only high- $Z$ star that is inconsistent in its Fourier parameters is V714 Cyg. Its value of $R_{21}=0.41$ is appropriate to an AHB1 star, while in the $\phi_{31}$ versus period diagram it is among the AHB2 stars. In the $\phi_{31}$ versus $\phi_{21}$ diagram V714 Cyg falls close to the sequence of the AHB1 and AHB3 stars. We considered this star to be an AHB2 star on the basis of the 
bump during rising light. However, looking at the light curve (Figure $1 d$ ), it can be seen that the bump is much weaker than for typical AHB2 stars like V971 Aql and VZ Aql (both in Figure $1 c$ ). Furthermore, the narrow maximum and bump during decline make this light curve unlike any other in our sample. Of the AHB2 stars observed by Schmidt et al. (2003a), only V714 Cyg lacks any indication of emission at $\mathrm{H} \alpha$. This is clearly an interesting star that would be worth further investigation.

In each of the period-phase diagrams, Figures $2 d-2 f$, the low- $Z$ and high- $Z$ Cepheids appear to form a single, rather scattered, sequence. In the phase-phase diagrams, Figure 3 , it can be seen that the low- $Z$ stars populate the same region as the high- $Z$ stars. However, in the $\phi_{31}$ versus $\phi_{21}$ diagram, and to some extent in the $\phi_{41}$ versus $\phi_{31}$ plot, they also occupy an extended area toward lower values of $\phi_{21}$ and $\phi_{31}$, respectively. While stars that fall in this extended region are likely to be type I stars, in the region of overlap we cannot distinguish the type. Thus, we conclude that Fourier decomposition offers limited potential for helping to identify type II Cepheids among short-period stars.

\subsection{The Stability of the Pulsation}

\subsubsection{Changes in Light-Curve Shape}

Excessive scatter is evident in a number of the light curves in Figure 1. In some cases an examination of the various data sets shows that this scatter is due to long-term changes in the lightcurve shapes. For these stars we have used different symbols to distinguish different sets of data, and we discuss them individually here.

\subsubsection{MQ Aql}

For MQ Aql, Figure $1 b$, we have plotted the present photometry as circles and Henden's (1996) photometry as plus signs. The Henden points are fainter than ours by between 0.2 and 0.35 mag during declining light. While we have adjusted our data to match the zero point of older photometry in some cases, this is an implausibly large adjustment. Additionally, the slope of declining light differs noticeably between the two data sets. We conclude that the light-curve shape for MQ Aql has changed between the observations of Henden and the present observations.

Henden's data were scattered over an interval of more than 2200 cycles, while all but two of our data points were obtained during 275 cycles. Thus, it is surprising that the scatter in Henden's light curve is noticeably less than that in ours. This may suggest that short-term changes occur in the light curve.

\subsubsection{SW Tau}

For SW Tau, Figure $1 b$, we have plotted our data as circles and the data of Henden (1980) as crosses. The remainder of the photometry, from Szabados (1977), Moffett \& Barnes (1984), and Barnes et al. (1997), is represented by plus signs. Over most of the cycle, there is little scatter. Around maximum light our data and that of Henden fall significantly above the mean curve. The fact that the Szabados data cover an interval of 900 days before the Henden data and the Moffet \& Barnes and Barnes et al. data cover an interval of 2200 days between Henden's observations and ours might cast some doubt on the reality of any light-curve changes. On the other hand, of the nine points around maximum from our data and that of Henden, only two fall on the mean curve. This and the fact that these data were obtained on seven different nights tends to strengthen the conclusion that the light curve occasionally changes around maximum light.

A further peculiarity in the SW Tau data is the excess scatter around the middle of declining light and around minimum. The deviant points come from several of the data sets. This and the fact that it occurs in rather restricted ranges of phase argue for its reality. However, any firm conclusion about this scatter and that at maximum must be considered tentative until confirmed by further observations.

\subsubsection{V745 Oph}

In the light curve of V745 Oph, Figure $1 c$, the plus signs represent the data from Kwee \& Diethelm (1984), and the circles represent our data. As noted above these two data sets are phased with different ephemerides. It can be seen that there is very little difference in the light curves except perhaps during declining light and just after maximum. The light curve is quite stable in spite of the period change.

$$
\text { 4.2.1.4. BB Gem }
$$

The photometry of Szabados (1977; plotted as plus signs in Figure 1e) is systematically brighter during declining light and declines less rapidly than the present data and that of Henden (1980) (both plotted as circles). As a result, the amplitude is smaller. This is unlikely to be due to photometric error and probably represents a bona fide change in the light curve.

\subsubsection{V465 Oph}

In the phase plot of V465 Oph, Figure $1 f$, plus signs represent the data of Kwee \& Diethelm (1984), and circles represent the present photometry. We have determined the period that produces the best match of the light curve during declining light. This results in the rising branch of the Kwee \& Diethelm data proceeding that in our data by approximately 0.03 cycles. There are also differences between the light curves around minimum and maximum light. Thus, while the change in the light curve is smaller than in the other stars, it appears to be genuine.

$$
\text { 4.2.1.6. Summary }
$$

Of the four stars that exhibit light-curve changes (MQ Aql, SW Tau, BB Gem, and V465 Oph), two are high- $Z$ stars, and two are low- $Z$ stars. The Diethelm classes of these stars include one $\mathrm{AHB} 1$, one $\mathrm{AHB} 3$, one $\mathrm{C} \delta$, and one that may be $\mathrm{AHB} 3$ or $\mathrm{C} \delta$. Three are classed as type II Cepheids, CWB, in the GCVS, while the fourth is classified as a classical Cepheid, DCEP. Thus, these objects have nothing obvious in common. An understanding of the significance of the changes in the light curves must await a larger sample of objects or further information on these stars.

\subsubsection{Period Changes}

In Paper I we used the quantity $\Delta P$, listed in column (4) of Table 3, as a measure of long-term period stability. It is a less sensitive measure of period variations than is afforded by the construction of an $\mathrm{O}-\mathrm{C}$ diagram and is more subject to statistical vagaries. However, it can be determined for stars with a limited observational history.

Szabados $(1977,1991)$ studied the period changes of four of our stars, SW Tau, BL Her, BB Gem, and V351 Cep. In the first paper he found that the $O-C$ diagram of SW Tau could be accounted for by two intervals of constant period with a change of -0.000039 days between them. This is comparable to our value of $\Delta P=-0.000026 \pm 0.000005$ days. In the second paper he fitted the $O-C$ residuals with a continuously decreasing period. In that case, our value of $\Delta P$ corresponds to the change in period expected over about 16 yr. For BL Her, Szabados (1977) inferred one period change of -0.0001 days, which is of the same sign but larger in absolute value than our value, $\Delta P=$ $-0.000008 \pm 0.000002$ days. It is possible that the observations 
we used did not happen to include a large period change. For BB Gem, Szabados found a constant period; this is consistent with our negligible value of $\Delta P$. Finally, for V351 Cep, Szabados (1991) found a constant period. However, Erleksova (1978) studied the period behavior of this star over an earlier interval and found two period changes comparable to our value of $\Delta P$ and of the same sign. We conclude that while $\Delta P$ has its shortcomings it provides useful information on the stability of the periods of pulsating stars.

Wehlau \& Bohlender (1982) measured period changes for Cepheids in several globular clusters. Of the 10 stars on their list with periods shorter than 3 days, six exhibited increasing periods, while the values for the other four were smaller than the uncertainty. Two of the stars in $\omega$ Cen were later studied by Gonzalez (1994), who obtained a period change rate for one star, V43, which agreed with Wehlau \& Bohlender. However, for the other, V92, Gonzalez found that the period was decreasing at a large rate, -56 day $\mathrm{Myr}^{-1}$, as opposed to the sizable increase found by Wehlau \& Bohlender, 11.32 day $\mathrm{Myr}^{-1}$. Gonzalez attributed the difference to his handling of the uncertainty introduced by the cycle count. Regardless of the status of this star, it is clear that increasing periods predominate among Cepheids in globular clusters.

In looking at Table 3 it is obvious that a large majority of the stars have increasing periods. If we restrict our attention to the high- $Z$ stars, we find that nine have positive values of $\Delta P$, four have values less than the uncertainties, and four have negative values. Thus, our results show that the field type II Cepheids resemble the globular cluster Cepheids in having predominantly increasing periods. We note that Wehlau \& Froelich (1994) showed that there were more nearly equal numbers of increasing and decreasing periods among the RR Lyrae stars. They interpret this to show that while the RR Lyrae stars are probably affected by period fluctuations, the systematic trend among the Cepheids indicates that the period changes are evolutionary. They also show that the magnitude of the changes are reasonable compared with evolution theory.

We have plotted $|\Delta P|$ against the period in the top panel of Figure 4. The error bars are from column (3) of Table 3; most of them are smaller than the plotted points. The majority of the points lie along the bottom of the plot; 20 of the 24 stars have $|\Delta P|<0.00015$ days with an average of 0.000032 days. This is in contrast to the situation among the long-period stars (Fig. 3a of Paper I) for which we drew the line between the bulk of the points and those that were judged to be abnormally high at $|\Delta P|=0.015$ days. The average for these stars was $|\Delta P|=$ 0.0047 days. We also note that the largest value of $|\Delta P|$ in Figure 4 is almost 100 times smaller than the largest value found among the long-period stars. Thus, the period changes are about 2 orders of magnitude smaller in the short-period stars than in the long-period stars. Even the relative period changes, $|\Delta P| / P$, are an order of magnitude smaller in the short-period stars.

Looking first at the majority of the stars that fall along the bottom of the plot, it appears that the period stability of the high- $Z$ and the low- $Z$ stars are very similar. Leaving out the longest period star, BC Aql, the average for the low- $Z$ stars is 0.000028 days, as compared with 0.000026 days for the high- $Z$ stars. Nor is there any apparent segregation related to the light-curve types. This suggests that most short-period Cepheids, regardless of whether they are type I or type II and regardless of the type of the light curve, share the same degree of period stability.

In Figure 4, four stars stand out as having exceptionally large period changes. Three of them, V745 Oph, EK Del, and V465 Oph, are high- $Z$ stars. The fourth, V351 Cep, is listed in the
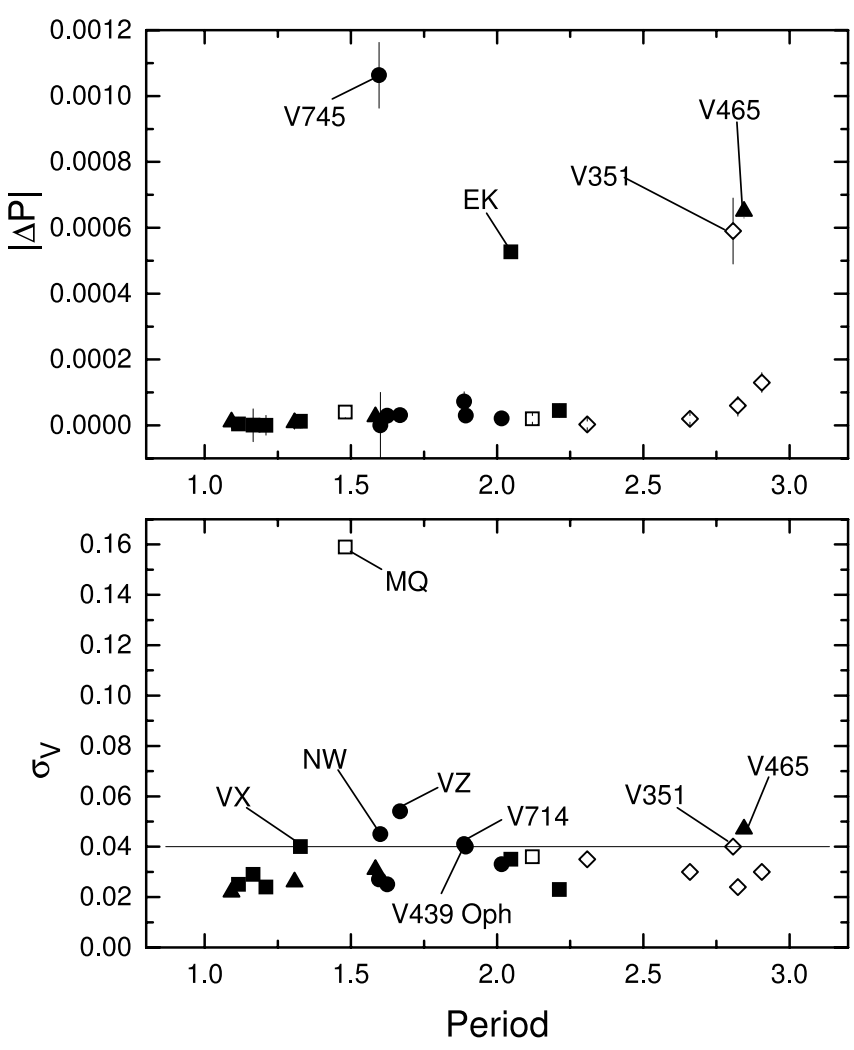

FIG. 4.- Period stability parameters plotted against period. The symbols have the same meaning as in Fig. 2. A number of stars that are relevant to the discussion in the text are identified.

GCVS as a CWB. However, Diethelm (1990) regarded it as an s-Cepheid (light-curve type SA), which is supported by its location in Figure 3. However, the scatter in the light curve is not typical of s-Cepheids (e.g., see the light curves shown in Mantegazza \& Poretti 1992). Erleksova (1978) noted several similarities between V351 Cep and the peculiar Cepheid AU Peg, including the large period changes. There has been disagreement as to whether AU Peg is a type I or type II Cepheid (see Szabados 1991 and Vinko et al. 1993 for a discussion), and the same uncertainties may apply to V351 Cep.

From this discussion we conclude that most stars in this period range have rather stable periods with only small long-term changes. The few exceptions seem to be dominated by high- $Z$, presumably type II, Cepheids. V351 Cep is an interesting exception and should be investigated further.

\subsubsection{Light-Curve Scatter}

The light-curve scatter, $\sigma_{V}$, is plotted against period in the bottom panel of Figure 4. The large value for MQ Aql is mostly due to the light-curve change discussed above. However, even if we consider only the data of Henden (1996), we get a value of $0.060 \mathrm{mag}$, which is higher than that of any other star. Aside from MQ Aql, all of the low- $Z$ stars have values of $\sigma_{V} \leq$ $0.040 \mathrm{mag}$. On the other hand, about one-third of the high- $Z$ stars have $\sigma_{V} \geq 0.040 \mathrm{mag}$. This is in contrast to the longperiod stars in which three-fourths of the high- $Z$ stars exhibited scatter greater than $0.045 \mathrm{mag}$ and three-fourths of the low- $Z$ stars fell below that value. Again, we find that the pulsation is more stable among the short-period high- $Z$ stars than among the long-period high- $Z$ stars.

The only low- $Z$ star with large scatter is MQ Aql. However, it exhibits doubling in the absorption core of $\mathrm{H} \alpha$ (Schmidt et al. 
2003b) and has an AHB1 light curve. It is likely to be a lowlatitude type II Cepheid. This being the case, the presence of increased scatter in the light curve is a strong indication that a star is a type II Cepheid. Unfortunately, the converse is not true, since most type II Cepheids appear to show little scatter. Lightcurve scatter is of limited usefulness in distinguishing type I from type II Cepheids in the short-period range.

The referee noted that the amplitude, shape, and scatter of the light curve of V351 Cep resemble those of double-mode Cepheids. We searched our photometry for another period near 2 or 4 days (assuming that the 2.8 day period is either the fundamental or the first overtone, respectively). No periodicity was detected near either period. Although this could be due to the coincidence of these periods with an integral number of days, it seems unlikely that this star is a double-mode pulsator.

\section{CONCLUSIONS}

Assuming that the high- $Z$ stars are predominantly type II Cepheids and the low- $Z$ stars are predominantly type I Cepheids, several conclusions can be drawn from the above discussion.

1. The Fourier parameters of the light curves distinguish among the three light-curve types of type II Cepheids, AHB1, $\mathrm{AHB} 2$, and $\mathrm{ABH} 3$, but are of limited usefulness in distinguishing between type I and type II Cepheids.

2. The stability of pulsation in the short-period Cepheids was investigated through long-term changes in the light-curve shape, period changes, and light-curve scatter. In each of these parameters, several stars stood out as having less stable pulsation. However, for the most part, different stars were identified by each parameter.

3 . The pulsation is considerably more stable among shortperiod stars than among long-period stars. This is to be expected if the scatter in type II Cepheids represents the onset of chaotic behavior as suggested by Kovacs \& Buchler (1988).

No one photometric parameter seems to be able to distinguish between type I and type II Cepheids in this period range. However, the partial discrimination provided by each of the parameters discussed here suggests that a combination of parameters may be successful in separating the two types of stars. After we complete the analysis of the photometric data for the intermediate period stars, we plan to combine all of the data and seek ways to combine the various observational parameters to reliably distinguish the two groups.

The referee of this paper made a number of suggestions that have significantly improved the discussion. We are grateful to him or her for a careful reading of the manuscript. We made extensive use of the McMaster Cepheid Photometry and Radial Velocity Data Archive in both the selection of stars for the program and in locating photometric data for use in this paper. We are appreciative of the efforts on the part of Douglas Welch in providing this resource. This material is based on work supported by the National Science Foundation under grant AST 00-97353.
Alcock, C., et al. 1999, ApJ, 511, 185

Antonello, E., \& Poretti, E. 1986, A\&A, 169, 149

Antonello, E., Poretti, E., \& Reduzzi, L. 1990, A\&A, 236, 138

Barnes, T. F., Fernley, J. A., Frueh, M. L., Navas, J. G., \& Moffett, T. J. 1997, PASP, 109, 645

Berdnikov, L. N. 1987, Perem. Zvezdy, 22, 530

1992a, A\&A Trans., 2, 31

1992b, A\&A Trans., 2, 43

1992c, A\&A Trans., 2, 107

1992d, A\&A Trans., 2, 157

1992e, Pis'ma Astron. J., 18, 325

1993, Pis'ma Astron. J., 19, 210

Diethelm, R. 1983, A\&A, 124, 108

1986, A\&AS, 64, 261

1990, A\&A, 239, 186

Diethelm, R., \& Tammann, G. 1982, A\&AS, 47, 335

Erleksova, G. E. 1978, Inf. Bull. Variable Stars, 1460

Gonzalez, G. 1994, AJ, 108, 1312

Harris, H. C. 1980, Ph.D. thesis, Univ. Washington

1985, AJ, 90, 756

Harris, H. C., \& Wallerstein, G. 1984, AJ, 89, 379

Henden, A. A. 1980, MNRAS, 192, 621

$$
\text { 1996, AJ, 112, } 2757
$$

Kholopov, P. N., ed. 1985, General Catalogue of Variable Stars, Vols. 1 and 2 (4th ed.; Moscow: Nauka)

Nauka 1987, General Catalogue of Variable Stars, Vol. 3 (4th ed.; Moscow:

Kovacs, G., \& Buchler, J. R. 1988, ApJ, 334, 971

Kwee, K. K., \& Diethelm, R. 1984, A\&AS, 55, 77

Loomis, C. G., Schmidt, E. G., \& Simon, N. R. 1988, MNRAS, 235, 1059

Mantegazza, L., \& Poretti, E. 1992, A\&A, 261, 137

\section{REFERENCES}

Moffett, T. J., \& Barnes, T. F. 1984, ApJS, 55, 389 1985, ApJS, 58, 843

Pel, J. W. 1976, A\&AS, 24, 413

Petersen, J. O. 1986, A\&A, 170, 59

Petersen, J. O., \& Diethelm, R. 1986, A\&A, 156, 337

Poretti, E., \& Pardo, I. 1997, A\&A, 324, 133

Schmidt, E. G., Chab, J. R., \& Reiswig, D. E. 1995, AJ, 109, 1239

Schmidt, E. G., Johnston, D., Langan, S., \& Lee, K. M. 2004a, AJ, 128, 1748 (Paper I)

Schmidt, E. G., Johnston, D., Lee, K. M., Langan, S., Newman, P. R., \& Snedden, S. A. 2004b, AJ, 128, 2988

Schmidt, E. G., Langan, S., Lee, K. M., Johnston, D., Newman, P. R., \& Snedden, S. A. 2003a, AJ, 126, 2495

Schmidt, E. G., Lee, K. M., Johnston, D., Newman, P. R., \& Snedden, S. A. 2003b, AJ, 126, 906

Schmidt, E. G., \& Reiswig, D. E. 1993, AJ, 106, 2429

Schmidt, E. G., \& Seth, A. 1996, AJ, 112, 2769

Simon, N. R. 1986, ApJ, 311, 305

Simon, N. R., \& Lee, A. S. 1981, ApJ, 248, 291

Smith, H. A., Silbermann, N. A., Baird, S. R., \& Graham, J. A. 1992, AJ, 104,1430

Sturch, C. R. 1966, PASP, 78, 210

Szabados, L. 1977, Mitt. Sternw. Akad. Wiss., 70 1991, Comm. Konkoly Obs., 96, 123

Udalski, A., Soszynski, I., Szymanski, M., Kubiak, M., Pietrzynski, G., Wozniak, P., \& Zebrun, K. 1999a, Acta Astron., 49, 1 1999b, Acta Astron., 49, 437

Vinko, J., Szabados, L., \& Szatmary, K. 1993, A\&A, 279, 410

Wehlau, A., \& Bohlender, D. 1982, AJ, 87, 780

Wehlau, A., \& Froelich, N. 1994, AJ, 108, 134 